\title{
Design for 3D Printing: case study for a cold plastic deformation mould
}

\author{
Andrei-Bogdan Nuță ${ }^{1, *}$, Mihaela-Elena Ulmeanu ${ }^{1}$, and Cristian-Vasile Doicin ${ }^{1}$ \\ ${ }^{1}$ University POLITEHNICA of Bucharest, Splaiul Independentei 313, Bucharest, Romania
}

\begin{abstract}
Integration of additive manufacturing throughout a product's lifecycle has proven over the years to bring substantial competitive advantages to companies worldwide. Complex geometries, quick iteration and lead-time reduction are universally seen as the biggest benefits of 3D printing. North American users also cite cost savings as a major benefit. More than half of the technologies' applications are related to prototype manufacturing, especially due to high-cost savings in the development phase. Complex prototypes often require a cross reference when it comes to the design rules which need to be considered during the development stage. Thus, this study aims to analyze the various parameters when designing and manufacturing a complex prototype using material extrusion. Some of the main issues covered are related to analyzing the interference between components, adjusting the dimensions of the component elements according to the material contractions, the amount of used material and the total scrap and costs. In order to evaluate the abovementioned, a case study for a cold plastic deformation mould was chosen. The components were designed and assembled in a 3D software after which, each part was exported in *.STL and *.Gcode formats. Assembly tests were performed on the 3D printed components in order to adjust the dimensions. Project planning was used to propose an accurate time frame for the final complex prototype. Cost evaluation and material consumption were discussed in relation to functional, technological and economical restrictions. A final budget and general design rules were proposed for $3 \mathrm{D}$ printing of the complex functional prototype.
\end{abstract}

\section{Introduction}

The purpose of this research is to create an entire cold plastic deformation mold and to achieve a set of parameters to obtain a complex product through additive manufacturing (AM) technologies, by material extrusion, and to analyze the points that require special attention. This mold has the functions of perforating, cutting and bending a piece of metal sheet. It is desired that the obtained prototype to be able to reproduce the same operations for a piece of cardboard paper. To date, there are no relevant experiments with cold plastic deformation molds $3 \mathrm{D}$ printed, all tests are for casting molds and in this case we are talking about materials from rigid to soluble.

\footnotetext{
*Corresponding author: andrei.bogdan12@gmail.com
} 
Considering the multitude of 3D printing technologies, according to Ma Quanjina et all, in the paper "Recent $3 D$ and $4 D$ intelligent printing technologies: A comparative review and future perspective" for this experiment any method of 3D printing in plastics could be used. FDM technology was chosen for ease, speed and low cost of printing [1]. Comparatively, if a technology such as SLA had been chosen, the parts would have been more accurate, but would have involved higher costs. Budget FDM printers and 3D printer kits start at a few hundred dollars. Higher quality mid-range desktop printers start around $\$ 2,000$, and industrial systems are available from $\$ 15,000$. Material cost is $\$ 50-\$ 150 / \mathrm{kg}$ for most standard and engineering filaments, and \$100-200/kg for support materials. And SLA Professional desktop printers start at $\$ 3,500$, large-format benchtop printers at $\$ 10,000$, and large-scale industrial machines are available from $\$ 80,000$. While material is $\$ 149-\$ 200 / \mathrm{L}$ for most standard and engineering resins. Taking into account the mentioned above, the plan that is wanted to be followed is to test the small assemblies, then the big ones to see which are the dimensional deviations that must be taken into account. Once the assemblies have been tested separately, the entire prototype is assembled.

Active elements of cold plastic deformation are already printed modularly and embraced by companies such as Wilson Tool International. They use interchangeable $3 \mathrm{D}$ printed active elements that are added to a metal support and can perform a multitude of tasks depending on demand. There are two ways to obtain the elements, buying a 3D printer to make the products in-house or buying the final elements directly. These elements are not suitable for applications that put a lot of force on the active elements. Their advantage is high adaptability at a low cost, about $\$ 20$ on average for an active element $[2,3,4]$. Proto G. printed on a Prusa i3 printer from PLA 12 tools to bend sheet of metal up to $3.4 \mathrm{~mm}$ thick. They had a $20 \%$ infill and were used to print over 150 pieces each. For a small - medium size press $80 \%$ of the applications are made with standardized tools and $20 \%$ with special tools that can be $3 \mathrm{D}$ printed to order [5]. This method of printing tools using additive technologies is called Rapid Tooling (RT). It is used both for printing active elements and for printing molds for polymeric materials. R. Udroiu [6] shows the differences in execution time and costs between obtaining an injection mold at low temperature by classical methods of silicone or ceramic) and obtaining it by $3 \mathrm{D}$ printing. He used polyjet technology with digital ABS as the material to make a mold for injecting resins much faster, more complex and cheaper.

Also the concept of Rapid Tooling is developed for the printing of metallic materials, where it can produce both injection and deformation molds and cutting tools. Emanuel Sachs et all, use binder jetting technology for development very precise parts [7]. All the tools were printed with 420 stainless steel powder and a bronze (90 copper, 10 tin) infiltrant. They manufacture molds for plastic injection, by high precision 3D printing and complex details. S. A. Jahan et all researched the optimization of cooling channels for such plastic injection molds [8]. Another great advantage of obtaining tools through additive printing technologies is the fact that we can apply a topological optimization on them and thus make large reductions in material and implicit weight where possible [9].

Technologies such as FDM can also be used to create both tools and auxiliary elements during processing such as jigs, fixtures, assembly aids, pulleys, tool holders, etc.. These parts help the worker a lot of time, help the repeatability of the process and reduce the waste being Poka-Yoke elements [10]. They can bring large increases in profit taking into account the fact that their manufacturing cost is low.

\section{Methods}

The project started with the analysis of the execution drawing of the mold, creating 3D model for each component and finally their assembly (Figure 1). 


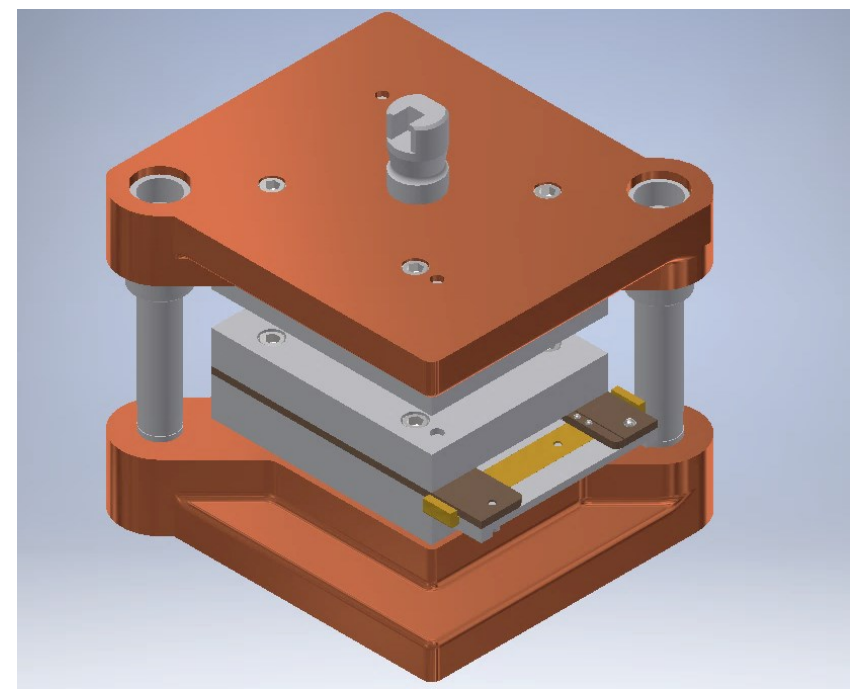

Fig. 1. 3D CAD Mold Assembly.

The next step was to save every component in high quality *.STL format. The *.STL format is the one recognized by 3D printing software, and their quality influences the resolution of the final part.

Printers used are from Zortrax company, they have their own software called Z-Suite which produces the Z-Code or printing process. The printers were Zortrax M200 and M300 with the dimensions of $200 \times 200 \times 185 \mathrm{~mm}$ and $300 \times 300 \times 300 \mathrm{~mm}$ printing space.

After the parts were saved in STL format, the Z-codes were generated for each. These codes that contain all the information of the future printed parts, the dimensions, the quality of the surfaces, the structure of the material, etc. The main stages through which parts were passed in the Z-Suite program for generating codes are described hereafter.

Choosing the type of printer used (M200 or M300), inserting the .STL file and positioning it on the printer's table (Figure 2). The most important aspect of this stage is the positioning of the piece. This is chosen according to the functional role, so that the functional surfaces can be printed correctly and we do no have the support structures here that can damage their quality.

Also depending on the forces that are applied on the pieces and their direction, the orientation of the layers is taken into account. According to Alkaios Bournias Varotsis [11] in the article "Introduction to FDM 3D printing" on 3dhubs.com, tensile strenght of a 3D printed part by FDM on the $\mathrm{Z}$ direction is always smaller than their strenght in the XY-plane. This resistance can be up to 4 times lower. 


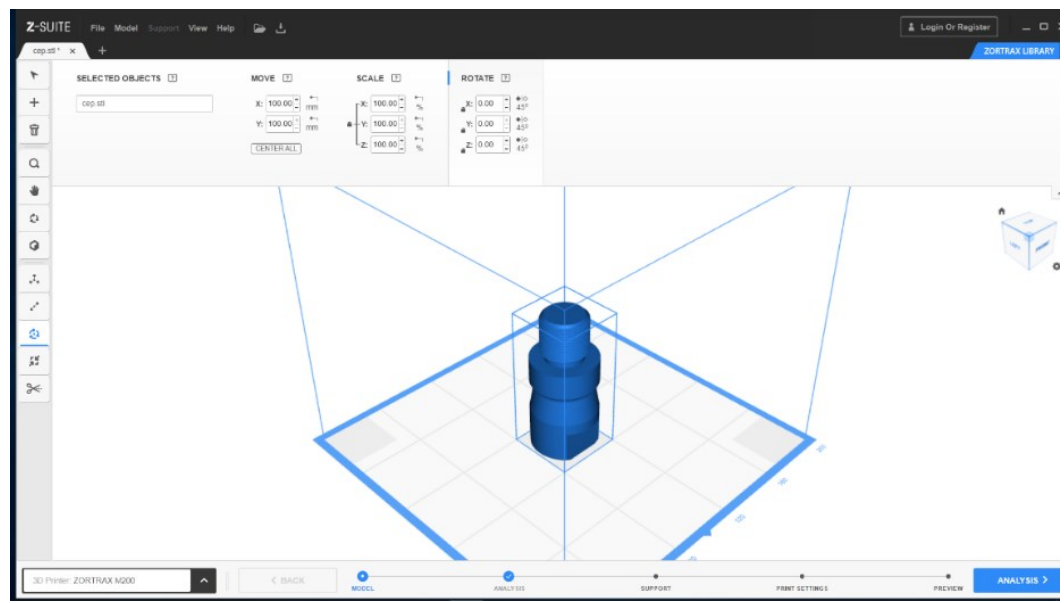

Fig. 2. Orientation of the part on the printer table.

The next step is to analyze the printer's ability to make all surafaces (Figure 3 ). As you can see, with green is the good area and with red are very thing surfaces that cannot be made correctly. Special attention has been paid to these surfaces. The printers used have a minimum resolution of $0.4 \mathrm{~mm}$ for walls, for this reason the margin of the thread appear in red being below this limit. For this experiment it was changed from threaded assembly to tight assembly in the post-processing stage.

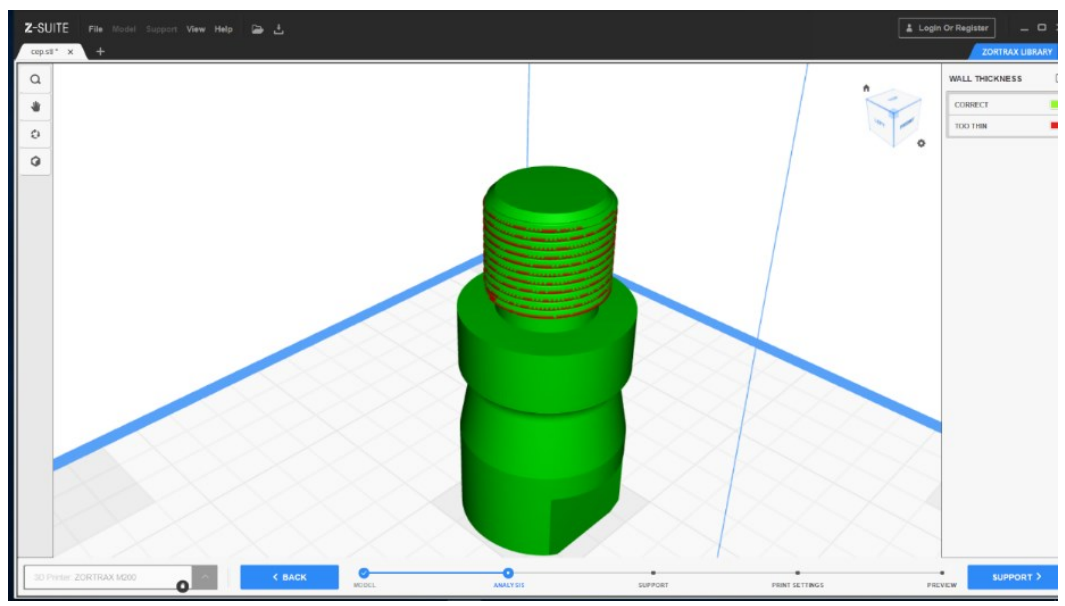

Fig. 3. Part verification in Z-Suite.

The next step is to add support structures and printing features. The support structure is added automatically or manually, the only thing to specify is to avoid adding large amounts of supports on surfaces that require a high finish. In figure 4 the piece is observed with blue color and with gray the support structure. According to Perry Cain [12] in the article "Supports in 3D Printing: A technology overview" on 3dhubs.com the support structure is required if the inclination is less than 45 degrees or if the distance between the bridge ends is more than $5 \mathrm{~mm}$. 


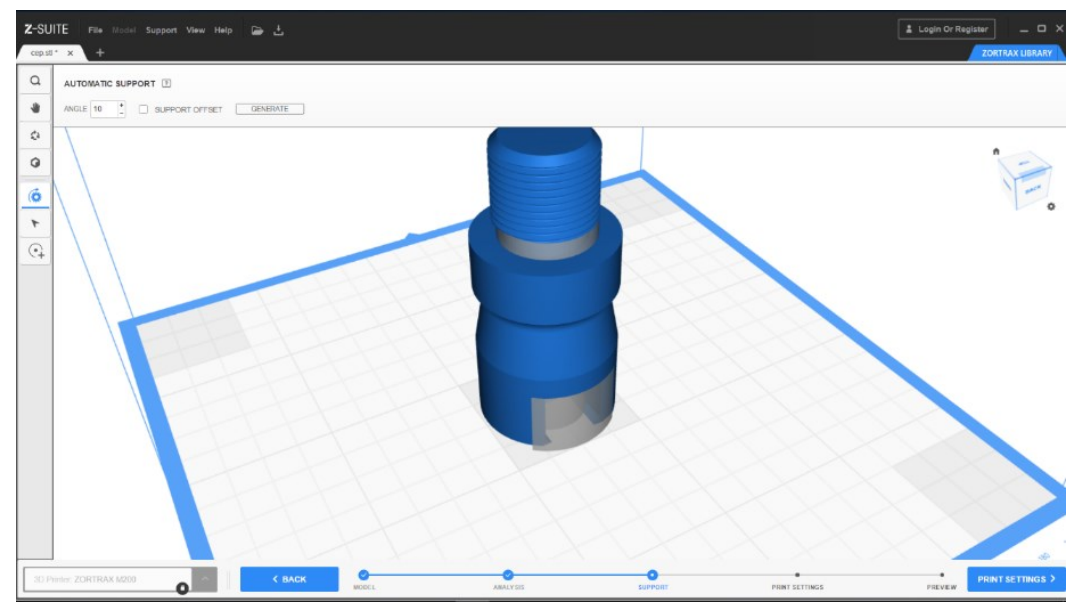

Fig. 4. Support structure in Z-Suite.

The most important step in making a Z-code is how the printing characteristics are chosen. the settings can be adapted according to the parameters, if a superior surface finish or a high resistance of the part or a low cost is desired. The settings chosen for the example part are visible in figure 5.

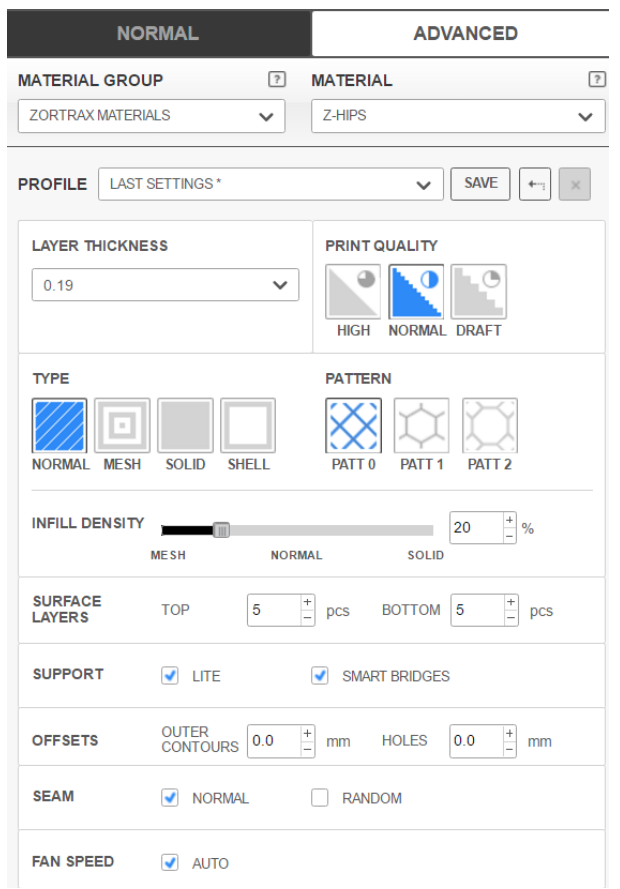

Fig. 5. Settings in Z-Suite.

In this case the material used for all components is Z-HIPS. A medium layer thickness of $0.19 \mathrm{~mm}$ was chosen. The quality of the surface in the conditions in which we do not have special restrictions is normal for time efficiency. The type of layer placement is normal. The interior model is PATT 0 rectangular. Infill density is set at $20 \%$ for low material consumption. The end complete layers are 5 to provide medium strenght. Similar settings 
were used for the other components. All the components fit entirely on the tables of the printers without the need to break them in pieces.

Next, the large elements were printed, after which the tests for the small elements that were assembled with them began. With the first test pins, at their native size, assembly was not possible. In order for the assembly to take place, their size has been reduced by 0.18 $0.25 \mathrm{~mm}$ in radius for parts samller than $20 \mathrm{~mm}$ diameter and with $0.4-0.5 \mathrm{~mm}$ for pieces over $20 \mathrm{~mm}$ diameter (Figure 6).

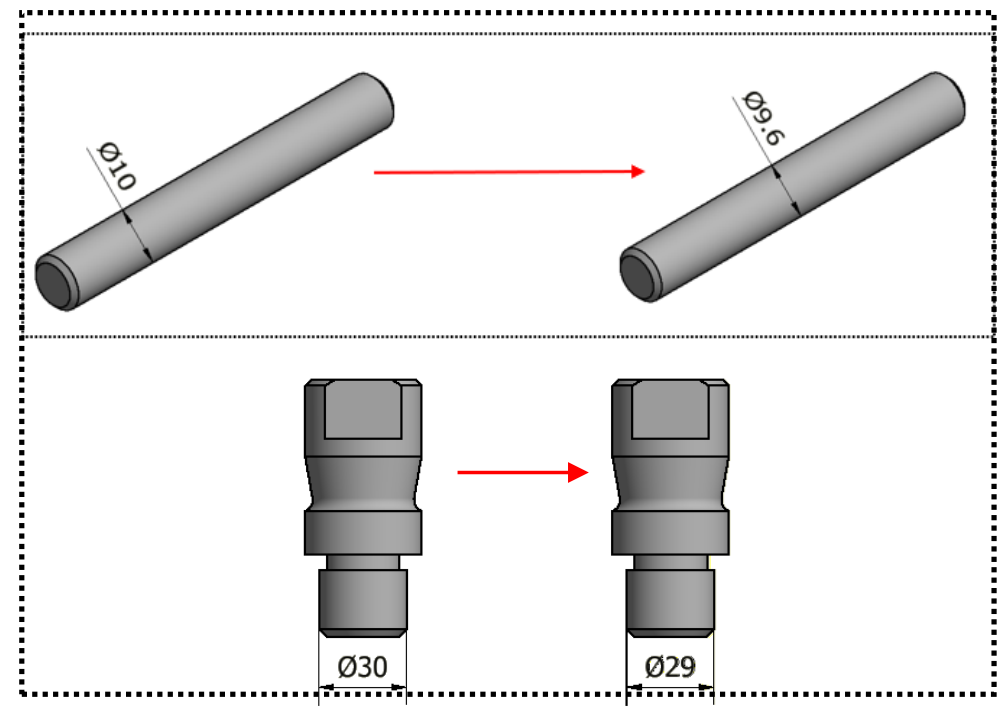

Fig. 6. Modification of cylindrical elements.

For piercers that do not have a cylindrical shape, the same reduction margins were used for each of their dimensions (Figure 7).

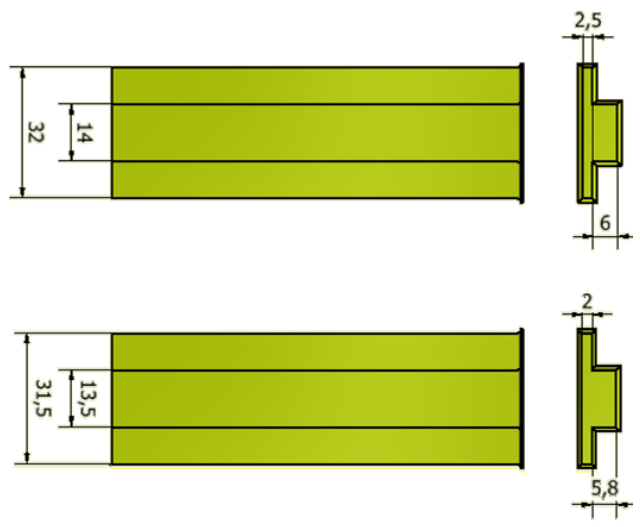

Fig. 7. Modification of non - cylindrical elements.

However, errors occurred in the threaded surfaces which were replaced by cylindrical surfaces and the assembly was performed by tightening. Threaded surfaces that did not print correctly were retouched by polishing (Figure 8). 


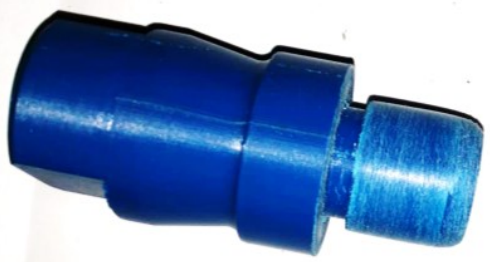

Fig. 8. Retouched part.

After applying the dimensional modifications for the column, bushing and end plate group, it was assembled correctly and functionally (Figure 9). To achieve this, the bushing was left unchanged, the diameter of the end plate was increased and the diameter of the column was reduced. Here is a fixed assembly and a mobile one.

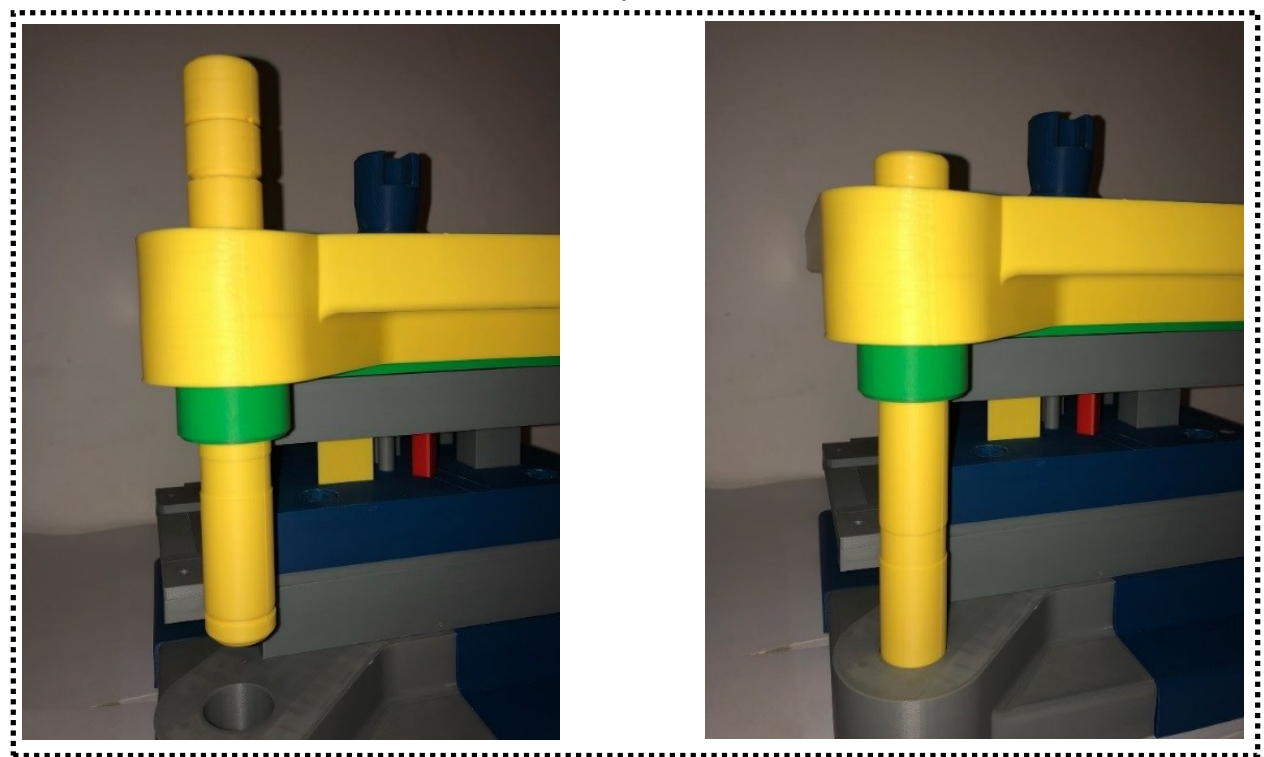

Fig. 9. Bushing - column - end plate assembly.

Other elements that are assembled are screws, pins and punches in different elements (Figure 10).

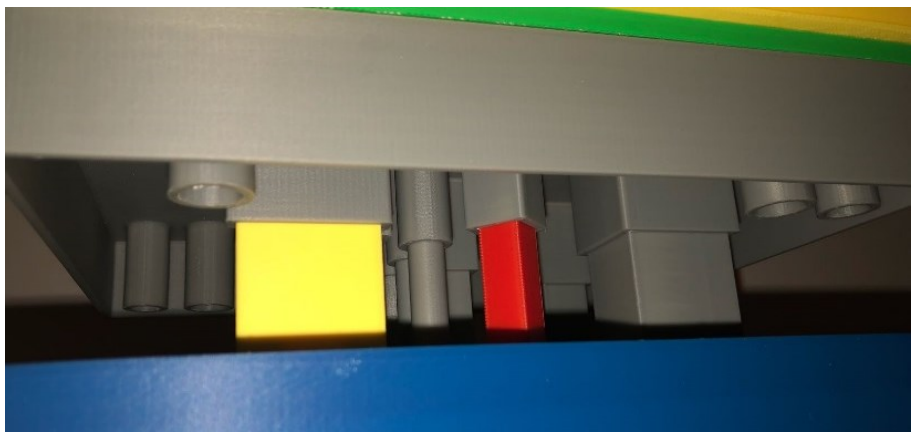

Fig. 10. Punches assembly 


\section{Results}

For this research, 3D printers from Zortrax were used, more precisely Zortrax M200 and M300.

Zortrax M200 specifications:

- Maximum printing space 200 x 200 x 185 mm;

- Filament diameter $1.75 \mathrm{~mm}$;

- Contains a single extruder head;

- $\quad$ Nozzle diameter $0.4 \mathrm{~mm}$;

- Overall dimensions fed with material 350 x 440 x $505 \mathrm{~mm}$;

- $\quad$ Layer resolution 90-390 microns;

- Minimum wall thickness 400 microns;

- Dimensional accuracy $+/-0.2 \%$;

- Angular accuracy $=/$ - $0.2 \%$;

- $\quad$ Software used Z-Suite;

- $\quad$ Maximum printing temperature $290^{\circ} \mathrm{C}$;

- Heated printing platform;

- $\quad$ Maximum platform temperature $105^{\circ} \mathrm{C}$;

- Ambient temperature $20^{\circ}-30^{\circ} \mathrm{C}$;

The material used to print the parts is Z-Hips (High impact polystyrene) with the following characteristics:

- $\quad$ Tensile strength $16.90 \mathrm{~N} / \mathrm{mm} 2$

- $\quad$ Breaking strength $13.02 \mathrm{~N} / \mathrm{mm} 2$

- $\quad$ Bending strength $29.30 \mathrm{~N} / \mathrm{mm} 2$

- Density $1,136 \mathrm{~g} / \mathrm{cm} 3$

- Melting point $200^{\circ} \mathrm{C}$

Table 1 shows the printing times and the quantities of material used for each part.

Table 1. Printing details

\begin{tabular}{|c|c|c|c|c|c|}
\hline $\begin{array}{l}\text { Nr. } \\
\text { Crt. }\end{array}$ & Designation & Amount & $\begin{array}{c}\text { Overall } \\
\text { dimensions }\end{array}$ & $\begin{array}{c}\text { Printing time / } \\
\text { piece }\end{array}$ & $\begin{array}{c}\text { Material } \\
\text { used/piece } \\
{[\mathrm{g}]}\end{array}$ \\
\hline 1 & Dowel & 1 & $\varnothing 40 \times 88$ & $2 \mathrm{~h} 49 \mathrm{~min}$ & 29 \\
\hline 2 & End plate & 1 & $293 \times 300 \times 55$ & 1d 10h $18 \mathrm{~min}$ & 556 \\
\hline 3 & Top package pin & 2 & $\varnothing 10 \times 75$ & $40 \mathrm{~min}$ & 4 \\
\hline 4 & Small bush & 1 & $\begin{array}{c}\varnothing 45 \times 72 \\
\text { Interior Ø30 }\end{array}$ & $3 \mathrm{~h} 5 \mathrm{~min}$ & 29 \\
\hline 5 & Big bush & 1 & $\begin{array}{c}\varnothing 45 \times 72 \\
\text { Interior Ø32 }\end{array}$ & $3 \mathrm{~h} 5 \mathrm{~min}$ & 29 \\
\hline 6 & Pressure plate & 1 & $160 \times 200 \times 5$ & $7 \mathrm{~h} 58 \mathrm{~min}$ & 108 \\
\hline 7 & Piercer plate & 1 & $160 \times 200 \times 30$ & $20 \mathrm{~h} 8 \mathrm{~min}$ & 190 \\
\hline 8 & Piercer 1 & 1 & $\varnothing 12 \times 105$ & $47 \mathrm{~min}$ & 4 \\
\hline 9 & Piercer 2 & 2 & $\varnothing 10 \times 105$ & $51 \mathrm{~min}$ & 4 \\
\hline 10 & Piercer 3 & 1 & $26 \times 8 \times 105$ & 1h 9min & 9 \\
\hline 11 & Piercer 4 & 1 & $34 \times 10.5 \times 105$ & $1 \mathrm{~h} 24 \mathrm{~min}$ & 12 \\
\hline 12 & Piercer 5 & 1 & $37.5 \times 18 \times 105$ & $2 \mathrm{~h} 14 \mathrm{~min}$ & 12 \\
\hline 13 & Piercer 6 & 1 & $\begin{array}{c}15.5 \times 15.5 \times \\
105\end{array}$ & $58 \mathrm{~min}$ & 8 \\
\hline 14 & Piercer 7 & 1 & $21.5 \times 11 \times 105$ & $1 \mathrm{~h} 16 \mathrm{~min}$ & 9 \\
\hline 15 & Piercer 8 & 1 & $32 \times 26 \times 105$ & $2 \mathrm{~h} 18 \mathrm{~min}$ & 24 \\
\hline 16 & Piercer 9 & 1 & $18 \times 17.5 \times 105$ & $1 \mathrm{~h} 15 \mathrm{~min}$ & 11 \\
\hline 17 & Guide plate & 1 & $200 \times 160 \times 30$ & $20 \mathrm{~h} 35 \mathrm{~min}$ & 194 \\
\hline
\end{tabular}




\begin{tabular}{|c|c|c|c|c|c|}
\hline 18 & $\begin{array}{c}\text { Semi-finished } \\
\text { guide plate }\end{array}$ & 2 & $145 \times 40 \times 6$ & $6 \mathrm{~h} 4 \mathrm{~min}$ & 78 \\
\hline 19 & Active plate & 1 & $200 \times 160 \times 35$ & $22 \mathrm{~h} 57 \mathrm{~min}$ & 209 \\
\hline 20 & Long upper pin & 2 & $\varnothing 10 \times 120$ & $1 \mathrm{~h} 3 \mathrm{~min}$ & 6 \\
\hline 21 & Short lower pin & 2 & $\varnothing 8 \times 35$ & $17 \mathrm{~min}$ & 2 \\
\hline 22 & Pusher & 1 & $177 \times 25 \times 12$ & $1 \mathrm{~h} 50 \mathrm{~min}$ & 22 \\
\hline 23 & Support plate & 1 & $160 \times 45 \times 11$ & $1 \mathrm{~h} 37 \mathrm{~min}$ & 21 \\
\hline 24 & Base plate & 1 & $300 \times 293 \times 60$ & $1 \mathrm{~d} 9 \mathrm{~h} \mathrm{53min}$ & 574 \\
\hline 25 & Small column & 1 & $\varnothing 30 \times 225$ & $4 \mathrm{~h} 26 \mathrm{~min}$ & 45 \\
\hline 26 & Big column & 1 & $\varnothing 32 \times 225$ & $4 \mathrm{~h} 26 \mathrm{~min}$ & 45 \\
\hline \multicolumn{7}{|c|}{ Total } & 7 d 5h 23min & 2250 \\
\hline
\end{tabular}

The printing time is 7 days 5 hours and 23 minutes for the final parts of the mold. Given the fact that we had a margin of rejection, so that not all the pieces came out of the first version or due to some technical problems the printing stopped, to this printing time is added 2 more days. Following this estimate, the total printing time is 9 days 5 hours and 23 minutes. The amount of material used for the mold elements is 2250 grams. Again, if we consider non-compliant scrap and parts, we consumed about 600 grams of material. In total there is a value of 2850 grams of Z-HIPS. The cost of this amount of material is about 185 euros. Taking into account that the equipment was not bought but those from the UPB faculty were used, the final cost is limited only to the material cost. The prototype for educational demonstration purposes cannot produce functional parts so the investment is the future example for students.

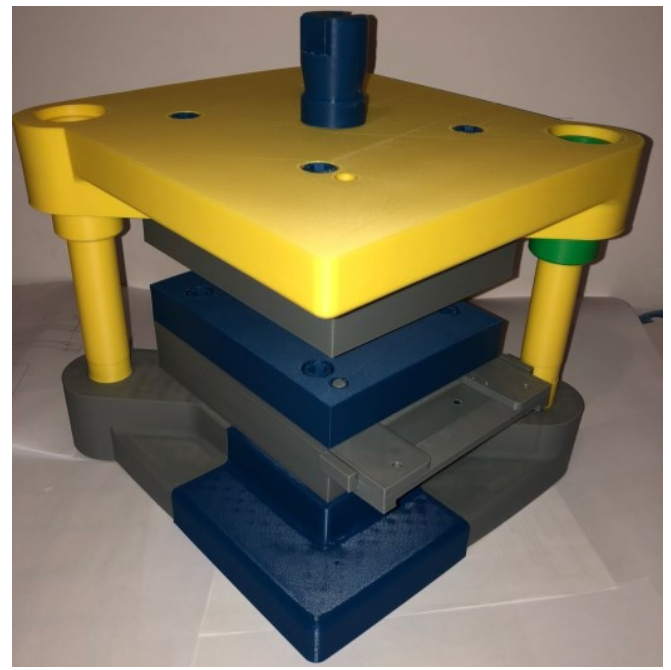

Fig. 11. Final mold assembled.

\section{Conclusions}

In the paper the process of obtaining files from the CAD model to the STL model and finally in Z-Code was detailed by using the Z-Suite software from Zortrax. As can be seen during the chapters, modifications were made to the CAD models for certain parts (punches, plates, columns), in order to improve the contraction of the material and finally to allow the assembly of components.

The printing parameters have been adapted according to each part and its dimensions to obtain the shortest possible printing time and for a minimum amount of material used even 
if the quality and strength of the parts has been affected. For example, the end plate and base plate parts have a low resistance to stress due to the low density of the inner layer and the large overall dimensions). In order for the assembly to take place, their size has been reduced by $0.18-0.25 \mathrm{~mm}$ in radius for parts samller than $20 \mathrm{~mm}$ diameter and with $0.4-0.5 \mathrm{~mm}$ for pieces over $20 \mathrm{~mm}$ diameter

Even with all these precautionary methods, not all parts fit after printing, and further processing was needed to reduce certain areas. We recorded the times and quantities of material used for each part as a whole and with these data we calculated the printing cost of the prototype. After printing, we tested the bushing - column, bushing - end plate, column base plate assemblies, pins - plates, punches - plates. Of these, the only problems occurred with punches - plates and dowel - end plate, where we intervened by reducing the size of the punches and dowel until the joint was appropriate.

Finally, all the elements were assembled to outline the final image of the mold, and the last changes were made for a better fit of the components.

\section{References}

1. M. Quanjina, M. R. M. Rejaba, M. S. Idrisa, N. M. Kumarc,M. H. Abdullaha, G. R. Reddyd, ICCIDS, Recent $3 D$ and $4 D$ intelligent printing technologies: A comparative review and future perspective, (2019)

2. T. Heston, Additive manufacturing's potential in low-tonnage bending, [Online]. Available : $\quad$ https://www.thefabricator.com/thefabricator/article/bending/additivemanufacturings-potential-in-low-tonnage-bending. [Accessed 10 April 2021]

3. D. Maloney, 3D-printed tools turn bench vise into expedient press brake, [Online]. Available: https://hackaday.com/2020/01/06/3d-printed-tools-turn-bench-vise-intoexpedient-press-brake/. [Accessed 12 April 2021]

4. T. Heston, Need a custom press brake tool? Try printing it, [Online]. Available: https://www.thefabricator.com/additivereport/article/additive/need-a-custom-pressbrake-tool-try-printing-it. [Accessed 15 April 2021]

5. G. Proto, 3D Printed Press Brake Forming Tools, [Online]. Available : https://www.instructables.com/3D-Printed-Press-Brake-Forming-Tools/. [Accessed 8 April 2021]

6. R. Udroiu, I. C. Braga, MATEC Web of Conferences 112, Polyjet technology applications for rapid tooling, (2017)

7. E. Sachs, S. Allen, H. Guo, J. Banos, M. Cima, J. Serdy, D. Brancazio, Massachusetts Institute of Technology , Progress on Tooling by 3D Printing; Conformal Cooling, Dimensional Control, Surface Finish and Hardness

8. S. A. Jahan, H. El-Mounayri, Optimal Conformal Cooling Channels in 3D Printed Dies for Plastic Injection Molding (2016)

9. N. Asnafi, J. Rajalampi, D. Aspenberg, A. Alveflo, Production Tools Made by Additive Manufacturing Through Laser-based Powder Bed Fusion (2020)

10. J. Hiemenz, 3D printing jigs, fixtures and other manufacturing tools (2017)

11. A. Bournias-Varotsis, Introduction to FDM 3D printing, [Online]. Available: https://www.3dhubs.com/knowledge-base/introduction-fdm-3d-printing/. [Accessed 8 March 2021]

12. P. Cain, Supports in 3D Printing: A technology overview, [Online]. Available: https://www.3dhubs.com/knowledge-base/supports-3d-printing-technology-overview/. [Accessed 09 March 2021] 